REVISTA PROYECCIONES N 11: 208-219

Julio 1986 - I.S.S.N. 0716-0917

\title{
CANTIDAD DE NODOS REQUERIDOS PARA FUNCIONES DE BASE EN DOS DIMENSIONES
}

Dr. HECTOR ROJO J.*

\section{INTRODUCCION.}

La mayor parte de los problemas de ingeniería no son lo suficien temente simples como para ser resueltos analíticamente, y por esta razón, métodos de aproximación numérica han sido siempre una herramienta necesaria iara los ingenieros y matemáticos. El método de los elementos finitos proporciona una poderosa y versátil técnica para resolver problemas de ingeniería que incluyen ecuaciones diferenciales en derivadas parciales. Aunque puede haber diversidad en la formulación del método de los elementos finitos, este método puede distinguirse por las siguientes características.

1. La idea fundamental del método es realizar una partición del

* Profesor del Departamento de Matemáticas, Facultad de Ciencias Básicas Universidad de Antofagasta. 
dominio de interés, en la mayor parte de los casos una región continua, en un número finito de subdominios que no se traslapan llamados elementos finitos y entonces definir el problema en cada uno de estos elementos.

2. Sobre cada uno de los elementos finitos las funcionales requeridas son aproximadas por medio de un selecto conjunto de funciones de base, lo cual es en realidad el paso más relevante del método.

3. Sustitución de las aproximaciones en las ecuaciones que gobiernan el problema, o en sus equivalentes, puesto que frecuentemente las ecuaciones que gobiernan el problema no son usadas directamente sino una formulación varacional es usada como alternativa.

4. Derivación de ciertas matrices individuales, llamadas matri ces de rigidez, en cada uno de los elementos finitos y superposición de to das ellas para obtener la matriz global de rigidez.

5. Generalmente el paso cuarto resulta en un sistema de ecuacio nes que debe ser resuelto para obtener los coeficientes de las funciones de base que aproximan a las funcionales requeridas.

Es interesante notar que la aproximación sobre un determinado la do del elemento finito depende, como se verá luego, de las funciones de ba se asociadas a ese lado y que éstas dependerán de ciertos puntos sobre el lado llamados puntos nodales.

CANTIDAD DE NODOS REQUERIDOS.

Para un elemento finito en particular, una base $\left\{w_{i}(x, y)\right\}$, $i=1,1, \ldots . m$ se dice de orden $n$ si para cada polinomio de grado $n$ existe un conjunto de escalares $\left\{a_{i}\right\}, i=1,2, \ldots, m$ tal que

$$
p_{n}(x, y)=\sum_{i=1}^{m} a_{i} w_{i}(x, y) .
$$


El máximo grado de los polinomios generados por las funciones de base es un factor crucial que afecta la exactitud y razón de convergencia en el método de los elementos finitos [McLeod, 1976, p. 245]. Una propiedad deseable en la aproximación vía elementos finitos es que las funciones de base garanticen a lo menos continuidad $C^{\circ}$ entre los elementos. Nos referimos a esta propiedad como conformidad de las funciones de base.

Las condiciones requeridas para que las funciones de base garanticen continuidad $\mathrm{C}^{\circ}$ entre los elementos son las siguientes:

1. Existe una función de base $w_{i}(x, y)$ asociada a cada nodo $i$.

2. La función de base $W_{i}(x, y)$ asociada con el nodo $i$ debe ser normalizada a la unidad en el nodo $i$.

3. La función de base $W_{i}(x, y)$ se anula en los lados opuestos al nodo $i \mathrm{y}$ en todos los nodos $j$ para $j \neq i$.

Por las condiciones descritas anteriormente, la aproximación vía elementos finitos sobre un particular lado de un elemento finito depende sólo de las funciones de base asociadas con los nodos ubicados sobre ese lado. Por esta razón, se necesitan tantos nodos sobre un determinado lado como grados de libertad tenga el polinomio de aproximación sobre ese lado.

Supongamos que el polinomio tiene grado $n$ y un lado del elemen to finito es una curva algebraica de grado $m$. El polinomio de grado $n$ tiene $(n+1)(n+2) / 2$ grados de libertad, como lo podemos ver en la figura 1.

El triångulo de Pascal en la figura 1 muestra que el número de elementos es

$1+2+3+4+\ldots \ldots+(n+1)=(n+1)(n+2) / 2$, 


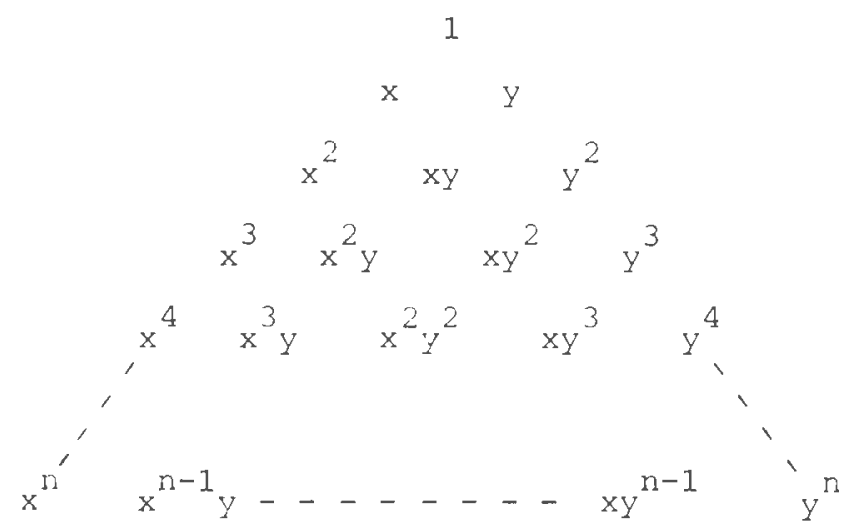

Figura 1. El triángulo de Pascal.

y $P_{n}(x, y)$ es generado por la base que contiene todos estos elementos.

[McLeod, 1977, p. 419] dice que para que la base genere polinomios de grado menor o igual que $n$ sobre una curva algebraica de grado $\mathrm{m}$ se necesitan $\mathrm{N}$ nodos sobre ese lado, donde

$N=\left[(n+1)(n+2)-u_{n m}(n-m+1)(n-m+2)\right] / 2$

$\operatorname{con} u_{n m}=1$ si $m \leqslant n ; O$ si m $>n$.

Una demostración interesante de esta afirmación consiste en probar que este número es la dimensión del espacio vectorial

$$
V=\left\{P_{n}(x, y): Q_{m}(x, y)=0, Q \text { irreducible }\right\} .
$$

Teorema.

$$
\operatorname{DimV}=[(n+1)(n+2)-(n-m+1)(n-m+2)] / 2, m \leqslant 1 .
$$

Demostración.

Inducción sobre m, n fija. 


$$
\begin{aligned}
& P_{n}(x, y) \text { es generado por el conjunto linealmente independiente } \\
& \left\{1, x, y, x y, x^{2}, y^{2}, \ldots . . x y^{n-1}, y^{n}\right\}
\end{aligned}
$$

$y$ esto implica que $\operatorname{dimP}_{n}=(n+1)(n+2) / 2$, entonces para todo

$P_{n}(x, y) \in P_{n}(x, y)$ existen $a_{i j} \in \mathbb{R}$ tal que

$$
P_{n}(x, y)=\sum_{i+j=0}^{n} a_{i j} x^{i} y^{j}
$$

1. Para $\mathrm{m}=1$,

$$
v_{I}=\left\{P_{n}(x, y): a x+b y+c=0\right\},
$$

donde a y b no son ambas nulas. Resolviendo para, digamos y, obtenemos $y=-(c+a x) / b, y$ por sustitución en $P_{n}(x, y)$,

$$
P_{n}(x, y)=\sum_{i+j=0}^{n} a_{i j} x^{i} y^{j}=\sum_{i+j=0}^{n} a_{i j} x^{i}(-(c+a x) / b)^{j}
$$

expresión que claramente es un polinomio de grado $n$ en la variable $x$, en tonces una base para $\mathrm{P}_{\mathrm{n}}(\mathrm{x}, \mathrm{y})$ es

$$
\left\{1, x, x^{2}, x^{3}, \ldots \ldots, x^{n}\right\}
$$

$y$ dimensión $v_{1}=(n+1)$.

$$
\begin{aligned}
& \text { También, para } m=1 \text { tenemos que } \\
& \begin{array}{l}
{[(n+1)(n+2)-(n-m+1)(n-m+2)] 2} \\
\quad=[(n+1)(n+2)-n(n+1)] / 2 \\
\quad=(n+1)(n+2-n) / 2=(n+1)
\end{array}
\end{aligned}
$$

2. Supongamos que 


$$
V_{k}=\left\{P_{n}(x, y): Q_{m}(x, y)=0\right\}
$$

es de dimensión

$$
\operatorname{dimv}_{k}=[(n+1)(n+2)-(n-m+1)(n-m+2)] / 2,
$$

entonces de $q_{k}(x, y) \in Q_{k}(x, y)$ tenemos que

$$
q_{k}(x, y)=\sum_{p+q=0}^{k} a_{p q} x^{p} y^{q}=0,
$$

donde no todos los coeficientes de los términos de grado $k$ son ceros. Podemos asumir sin pérdida de generalidad que el coeficiente $a_{o k}$ no es cero, entonces

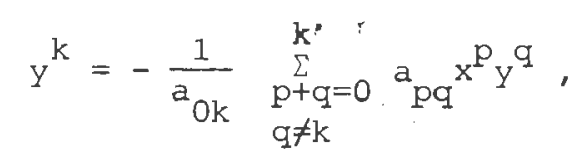

$y$ por sustitución en $p_{n}(x, y) \in P_{n}(x, y)$

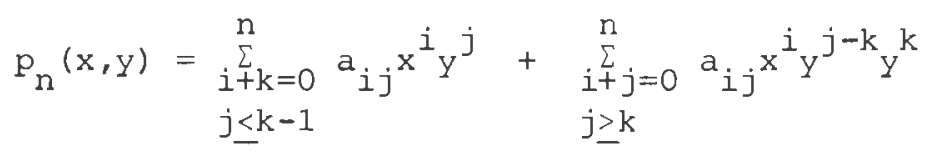

$$
\begin{aligned}
& =\sum_{\substack{i+j=0 \\
j \leq k-1}}^{n} a_{i j} x^{i} y^{j}+\sum_{\substack{i+j=0 \\
j \geq k}}^{n} a_{i j} x^{i} y^{j-k}\left(-\frac{1}{a_{0 k}} \underset{\substack{p+q=0 \\
q \neq k}}{k} a_{p q} x^{p} y^{q}\right)
\end{aligned}
$$

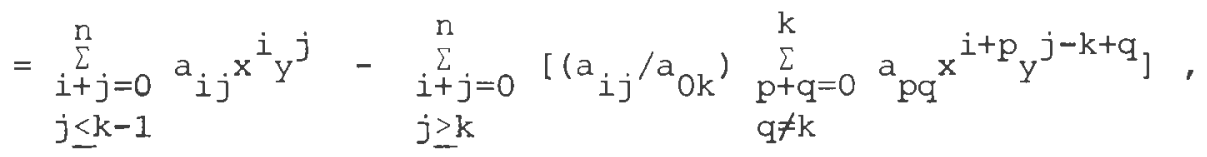

y de acuerdo a nuestra suposición

$$
\operatorname{dimv}_{k}=[(n+1)(n+2)-(n-k+1)(n-k+2)] / 2 .
$$

3. Sea ahora

$$
v_{k+1}=\left\{P_{n}(x, y): Q_{k+1}(x, y)=0\right\} .
$$


Resolviendo, sin pérdida de generalidad para $y^{k+1}$,

$$
y^{k+1}=-\frac{1}{a_{0}(k+1)} \sum_{\substack{p+q=0 \\ q \neq k+1}}^{k} a_{p q} x^{p} y^{q}
$$

$y$ por sustitución en $p_{n}(x, y) \in p_{n}(x, y)$

$$
\begin{aligned}
& p_{n}(x, y)=\sum_{\substack{i+j=0 \\
j \leq k}}^{n} a_{i j} x^{i} y^{j}+\sum_{\substack{i+j=0 \\
j \geq k+1}}^{n} a_{i j} x^{i} y^{j-k-1} y^{k+1} \\
& =\sum_{\substack{i+j=0 \\
j \leq k}}^{n} a_{i j} x^{i} y^{j}+\sum_{\substack{i+j=0 \\
j \geq k+1}}^{n} a_{i j} x^{i} y^{j-k-1}\left(-\frac{1}{a_{0}(k+1)} \underset{\substack{p+q \\
q \neq k+1}}{k+1} a_{p q} x^{p} y^{q}\right) .
\end{aligned}
$$

Pero

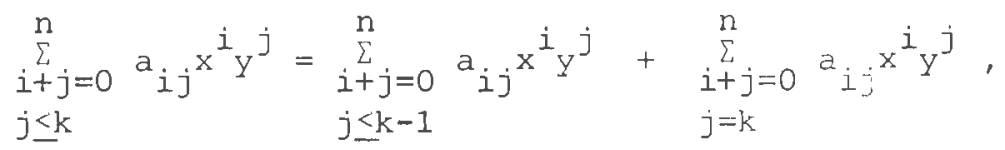

y

$$
\begin{aligned}
& \sum_{i+j=0}^{n}\left[\left(a_{i j} / a_{0}(k+1)\right) \sum_{\substack{p+q=0 \\
p \neq k+1}}^{k+1} a_{p q} x^{i+p} y j-k+q-i\right] \\
& j>k+1 \quad p \neq k+1 \\
& =\sum_{\substack{i+j=0 \\
j>k}}^{n}\left[\left(a_{i j} / a_{O k}\right) \sum_{\substack{p+q=0 \\
q \neq k}}^{k} a_{p q} x^{i+p} y y^{j-k+q}\right]
\end{aligned}
$$

entonces

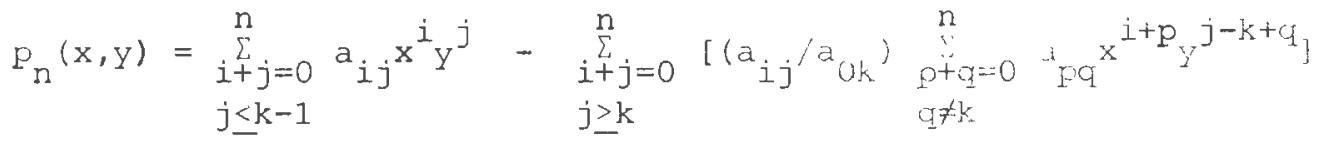

$$
\begin{aligned}
& +\sum_{\substack{i+j=0 \\
j=k}}^{n} a_{i j} x^{i} y^{j}
\end{aligned}
$$

Consideremos $p_{n}(x, y)=r(x, y)+s(x, y)$, donde 


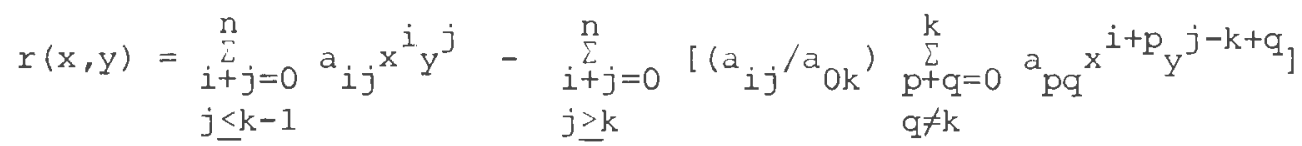

Y

$$
\begin{aligned}
& s(x, y)=\sum_{\substack{i+j=0 \\
j=k}}^{n} a_{i j} x^{i} y^{j} \\
& =a_{0 k} y^{k}+a_{1 k} x y^{k}+a_{2 k} x^{2} y^{k}+\ldots+a_{(n-k) k} x^{n-k} y^{k}
\end{aligned}
$$

De acuerdo a nuestra hipótesis de inducción, $r(x, y) \in v_{k}$ de dimensión $[(n+1)(n+2)-(n-m+1)(n-m+2)] / 2, y s(x, y)$ pertenece al subespacio $\bar{v}$ generado por $\left\{y^{k}, x y^{k}, x^{2} y^{k}, \ldots \ldots \ldots, x^{n-1} y^{k}\right\}$, es decir la dimensión de $\bar{V}$ es $(n-k+1)$. Notamos que $V$ puede ser escrito como la suma directa de los subespacios $V_{k}$ y $\bar{V}$, es decir, $V=V_{k} \oplus \bar{V}$; entonces

$$
\begin{aligned}
\operatorname{dimV}= & \operatorname{dimV} V_{k}+\operatorname{dim} \bar{V}, \text { or } \operatorname{dimV}=[(n+1)(n+2) \\
& -(n-k+1)(n-k+2)] / 2+(n-k+1) \\
= & {[(n+1)(n+2)-(n-k+1)(n-k+2)} \\
& +2(n-k+1)] / 2 \\
= & {[(n+1)(n+2)-(n-k+1)(n-k)] / 2 }
\end{aligned}
$$

lo cual completa la demostración.

Para regiones poligonales el triángulo y el rectángulo son los elementos finitos de uso más frecuente. El triängulo es probablemente el elemento finito de mayor uso y la razón es que polígonos arbitrarios pueden ser particionados exactamente en un número finito de regiones triangulares.

Consideremos una base de primer orden para un triángulo. Una 
función lineal tiene tres grados de libertad, pero sobre cada lado del triángulo, de acuerdo a (1) el número de graỉos de libertad se reduce a dos. De aquí que, para asegurar conformidad para la interpolación de lagrange se necesitan dos nodos sobre cada lado, los que son ubicados en los extrems de los ladus.

Sea $(i ; j)_{k}$ la forma lineul que pasic por los nodos $i y j$, con valor la unidad en el nodo k. Entonces las turciones de base para la aproxi mación lineal de Lagrange sobre el triängul, jur.

$$
w_{1}(x, y)=(2 ; 3)_{1}, \quad w_{2}^{\prime}(x, y)=(1 ; 3), w_{3}(x, y)=(1 ; 2)_{3} .
$$

Para una base de segundo orden para el triángulo, un polinomio cuadrático tiene seis grados de libertad, perc sobre cada lado del triángu 10, de acuerdo a (1), el número de gridus de ibertai se reduce a tres. Usualmente se usan nudos en ios trus vërt.es, " un nodo exträ en el punto, medio de cada ládo.

Las funciones de bast para li interpolación de Lagrange son:

$$
\begin{aligned}
& \left.w_{1}(x, y)=: 2 ; 3\right)(4 ; 1) \\
& w_{2}(x, y)=(1 ;),(4 ; 5) \\
& \left.w_{3}(x, y)=11 ;\right)_{3}(5 ;+) \\
& \left.w_{4}(x, y)=11 ; 3\right)_{4}(1 ; 1) \\
& w_{5}(x, y)=(1 ; 2) 5(3 ; 3) \\
& w_{6}(x, y)=(1 ; 2) 6(2 ;)
\end{aligned}
$$

Para el vaso cúbico, exister w! za ilez grados de iibertud los cuales se reducen a cuatro sobre cuáa I.ldi dei triángulo. Dos de ellos son localizados en los vërtices $y$ los intos dos en ios puntos de trisección de 
cada lado. Esto nos permite obtener sólo nueve nodos, de tal forma que se necesita un nodo extra, el cual usualmente es ubicado en el centroide del triángulo.

Las funciones de base para la interpolación cúbica de Lagrange son:

$$
\begin{aligned}
& w_{1}(x, y)=(2 ; 3)_{1}(5 ; 8)_{1}(4 ; 9)_{1} \\
& w_{4}(x, y)=(1 ; 3)_{4}(2 ; 3)_{4}(5 ; 8)_{4} \\
& w_{10}(x, y)=(1 ; 3)_{10}(1 ; 2)_{10}(2 ; 3)_{10}
\end{aligned}
$$

y similarmente para los otros seis nodos. Estas situaciones son mostradas en la figura 2 .
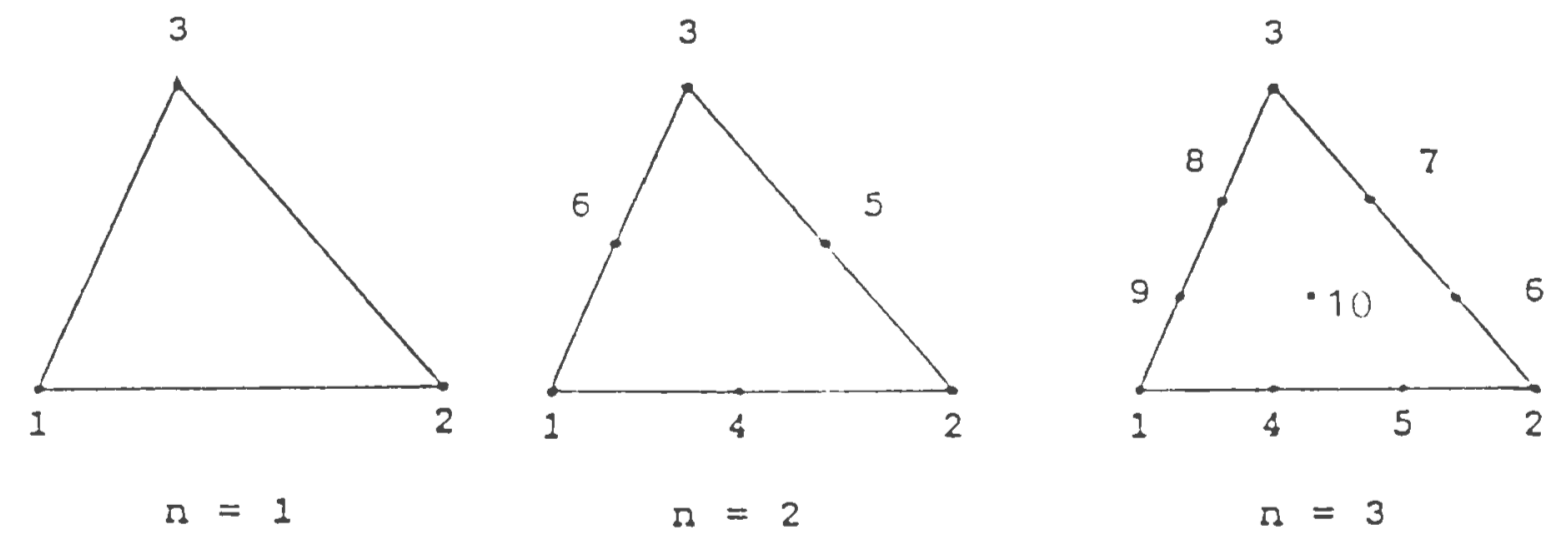

Figura 2. Nodos en un triângulo.

En el caso de una base de tercer orden usando la interpolación de Hermite sobre un triánguío, necesitamus interpolar la función y las pri meras derivadas parciales en cada vértice. Ta conformidad requiere que ca da función de base sea idénticamente cero sobre cada lado que no contiene su nodo. En un lado en particular, si el polinomio satisface las condicio nes de homogeneidad en los vértices, entonces esto significa que la corres pondiente curva algebraica tiene un punto de multiplicidad dos en los vértices. Esto implica que la curva tiene cuatro puntos en común con la linea que une los vértices, y de aquí que, por el teorema de Bézout [Primrose, 1955, p. 54], la curva y la línea tienen una componente en común. Pues 
to que la línea es irreducible, el polinomio debe ser cero en la línea.

Esto permite obtener un total de nueve funciones de base asociadas con los dobles nodos en los vértices. Puesto que un polinomio cúbico tiene diez grados de libertad, se necesita un nodo extra en el interior del triángulo y tomamos la interpolación da la función en ese punto.

Una generalización de esta situación a polinomios de grado menor - igual que $(2 n-1)$ puede ser encontrada en [McLeod, 1977, p. 103].

Cuando el dominio de interés del problema posee frontera curva, es frecuente aproximar la geometría de la región con elementos finitos triangulares que poseen un lado que es un arco cónico.

Para producir una base de segundo orden usando la interpolación de Lagrange en elementos triangulares con dos lados rectos y un lado que es un arco de una cónica, de acuerdo a (1), se necesitan tres nodos en cada lado recto y cinco nodos sobre el lado curvo del elemento, lo que resul ta en ocho nodos sobre este elemento de dos lados rectos $y$ un lado curvo.

Para el caso de una base de tercer orden sobre el mismo elemento, se requiere un total de doce nodos, siete ubicados en el arco cónico y cua tro en cada uno de los lados rectos. Esta situación es mostrada en la figura 3 .
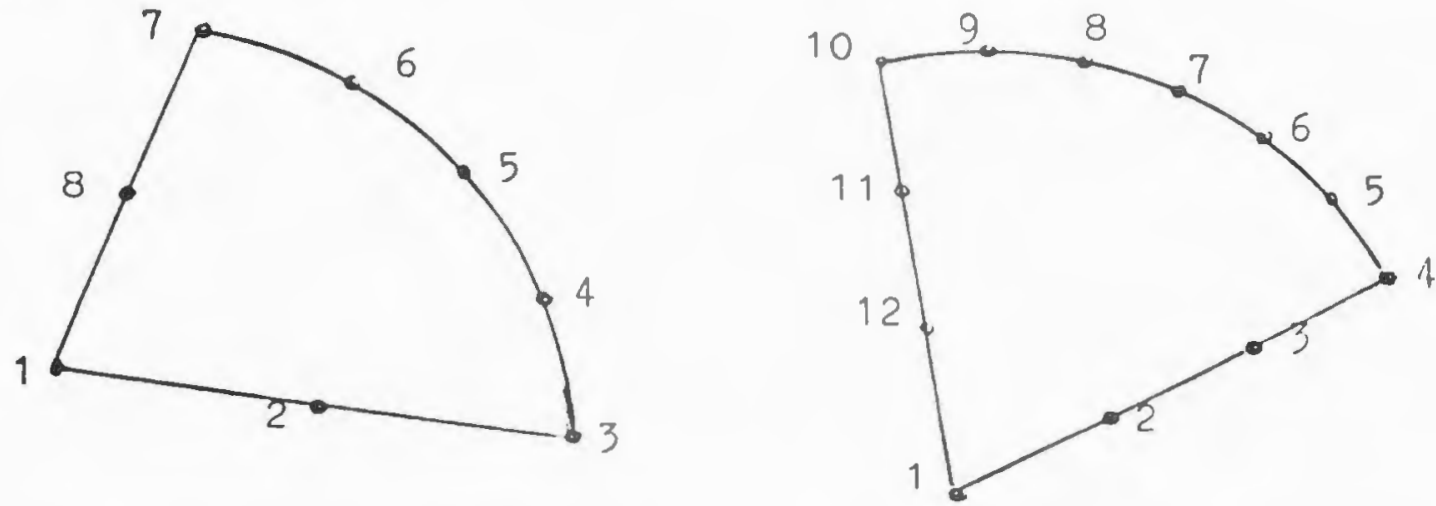

Figura 3. Posiciones nodales para bases de segundo y tercer orden respectivamente. 


\section{BIBLIOGRAFIA.}

(1) MCLEOD, R.J.Y. "Node Requirements for the High order Approximation Over Curved Finite Elements". J. Inst. Maths. Appl. 17(1976), 249-254 .

(2) MCLEOD, R.J.Y. "Hermite Interpolation Over Curved Finite Elements". J. Approx. Theory, 19(1977), 101-117.

(3) MCLEOD, R.J.Y. "High Order Transformation Method for Curved Finite Elements". J. Inst. Maths. Appl. 21(1978), 419-428.

(4) MITCHELL, A.R., WAIT, R. "The Finite Element Method in Parcial Diffe rential Equations". Willey and Sons, London, New York, Sydney, To ronto, 1977.

(5) PRIMROSE, E.J.F. "Plane Algebrac Curves". McMillan and Co., London, New York, 1955.

(6) ROJO, H.J. "Basis Functions in Curved Finite Elements". Doctoral Dissertation. New Mexico State University. 1985. 